\title{
Determining the Most Accurate and Early Form for Detection of Lyme Disease
}

\section{Samantha Zaremba}

\section{LYME DISEASE}

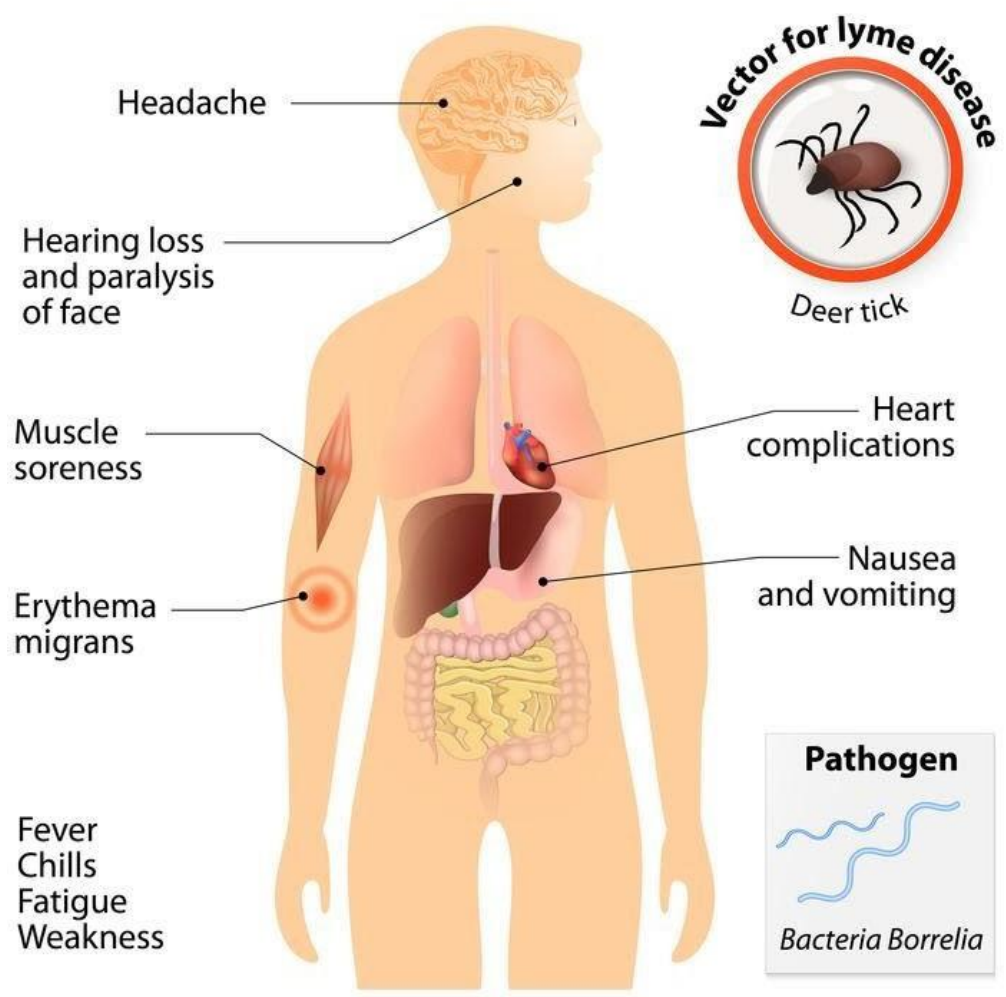

Source: independent, i.e.: https://universityhealthnews.com/daily/pain/lyme-disease/ 
Abstract

Lyme disease is the most common vector-based disease with over 300,000 new cases each year. The current diagnostic system for those with Early Lyme disease is only about $40 \%$ accurate. Since the severity of symptoms and probability of recurrence significantly increases as time progresses, there is a need to find a new alternative diagnostic system for Early Lyme disease. Many solutions to this problem are considered within this document, most notably: polymerase chain reaction detection, metabolic biosignature testing, and OspC targeting serological testing. After further analysis, metabolic biosignature testing is considered the best alternative since it yields the highest diagnosis sensitivity with around $89 \%$ accuracy. Additionally, metabolic biosignature testing is both cost effective and widely applicable since it does not require the Erythema migran rash to be used. Once in place, Lyme disease will be able to be diagnosed quicker, thus reducing the number of cases of Chronic Lyme disease which significantly reduces personal quality of life and is often costly.

\section{Keywords:}

Early Lyme disease, Metabolic Biosignature, Serological testing, Borrelia burgdorferi, Diagnostic Standards, Polymerase Chain Reaction

Document Scenario: This document analyzes the current diagnostic tests common for Early Stage Lyme disease. The aim of this proposal is to establish a more reliable and efficient test to diagnose Lyme disease during the early portions of infection. The target audience for this paper is the Center for Disease Control (CDC), the Department of Health and Human Services (HHS), and numerous other national and local health organizations with the mission of combatting infectious illnesses. This paper may be reviewed by regulation agencies such as the FDA to understand alternatives to the current symptomology and serologic tests being used to diagnose Lyme disease. 


\section{Executive Summary}

Lyme disease is a debilitating parasitic disease that causes a large range of non-specific symptoms including neurological and cardiovascular symptoms. With over 300,000 new cases of Lyme disease diagnosed every year, it is the seventh most common disease and the most common vector-based disease. While extremely common, diagnostic tests for Early Lyme disease are extremely inaccurate, with only an estimated $42 \%$ accuracy. Even worse, as Lyme disease progresses, symptoms grow significantly worse from initially mirroring common cold symptoms and a rash to chronic arthritis, muscle numbness, neurological deficiencies, and cardiovascular problems. However, with early detection and simple two-week treatment, chronic symptoms are rare. Consequently, a better alternative early diagnostic test is necessary so that the incidence of chronic symptoms is minimized.

An alternative diagnostic solution should have an accuracy more than double that of the current diagnostic solution that is able to selectively diagnose Lyme disease. Additionally, since time is of the essence, a testing system that is quick to perform is also a necessity. Finally, the test must cost less than the current system so that this diagnostic system is more attractive to hospitals and laboratories. While these are relatively easy goals for most bacterial diseases, Lyme disease is much harder to diagnose since there is only a very low concentration of the bacterial parasite that causes Lyme disease within the body. Additionally, while many bacteria can just be cultured, the incubation period for the Lyme disease parasite is around 21 days, making it extremely inefficient for diagnostic purposes. While over $60 \%$ of people experience some sort of rash from Lyme disease, the rash is extremely variable and can be misdiagnosed or overlooked. Additionally, not all patients experience this rash, so it cannot be used as a singular diagnostic factor.

The best alternative diagnostic test is metabolic biosignature testing. This relatively new diagnostic technology yields extremely high accuracy in the early stages of Lyme disease with a sensitivity of around $89 \%$. Additionally, the clinical sample is a noninvasive blood sample, similar to that of other Lyme disease diagnostic tests. As a singular blood test, costs for the analysis are only about a third of the price of the current Early Lyme disease diagnostic tests. 
Also, since the test only requires one blood test, the maximum time to perform such a test would be 3-5 days, which is typical for a blood test. Finally, since the test is a blood test, no rash is required for diagnostic testing, so the test can be performed on those with or without a rash.

Implementing this new diagnostic technique has extremely positive implications for those in areas where Lyme disease is epidemic. By making the diagnostic system for Lyme disease more accurate and less expensive, those who contract Lyme disease will be significantly less likely to experience lasting, chronic symptoms. This would not only improve the health of the nation, but also help save hospitals a lot of money that would have been spent treating those with Chronic Lyme disease. 
Maneto: The Temple University Multi-Disciplinary Undergraduate Research Journal | 3.1

\section{Table of Contents}

$\begin{array}{ll}\text { Executive Summary } & 3\end{array}$

$\begin{array}{ll}\text { Problem Analysis } & 5\end{array}$

Overview of problem and its significance $\quad 5$

Engineering fundamentals of problem $\quad 5$

Lessons from prior responses to the problem $\quad 8$

$\begin{array}{ll}\text { Project objectives and constraints } & 10\end{array}$

$\begin{array}{ll}\text { Candidate Solutions } & 11\end{array}$

$\begin{array}{ll}\text { Scope of solutions considered } & 11\end{array}$

$\begin{array}{ll}\text { Explanation of candidate solutions } & 11\end{array}$

Polymerase Chain Reaction (PCR) Detection 11

$\begin{array}{ll}\text { Metabolic Biosignature } & 13\end{array}$

Synthetic OspC based Serology 15

Comparative assessment of candidate solutions 16

$\begin{array}{lr}\text { Project Recommendations } & 18\end{array}$

$\begin{array}{ll}\text { Recommended solution } & 18\end{array}$

$\begin{array}{ll}\text { Design and implementation challenges } & 18\end{array}$

$\begin{array}{ll}\text { Implementation path } & 18\end{array}$

$\begin{array}{ll}\text { Possible Complications } & 19\end{array}$

$\begin{array}{ll}\text { Conclusion } & 19\end{array}$

$\begin{array}{ll}\text { Glossary } & 21\end{array}$

$\begin{array}{ll}\text { References } & 23\end{array}$ 
Maneto: The Temple University Multi-Disciplinary Undergraduate Research Journal | 3.1

\section{List of Figures}

Figure 1: Characteristics of Phylum Spirochaetes $\quad 6$

Figure 2: Different Forms of Erythema Migran Rash 9

Figure 3: System of Serological Tests Used in Diagnosing Lyme disease 10

Figure 4: Polymerase Chain Reaction Scheme 14

Figure 5: Binding System for Serological EIA Testing 17

\section{List of Tables}

Table 1: Effectivity of PCR testing of Various Clinical Specimen 15

Table 2: Comparative Analysis of Candidate Solutions 19 


\section{Problem Analysis}

This section reviews what Lyme disease is and why a new form of early detection is needed to help prevent chronic symptoms.

\section{Overview of problem and its significance}

Lyme disease is a tick-borne illness that is the result of microbial-sized parasites in the spirochete family called Borrelia burgdorferi $(\mathrm{Bb})$. Lyme disease is extremely common with over 300,000 new cases each year in the United States, which makes it the seventh most reported disease according to the US Centers for Disease Control and Prevention with over ninety percent of these cases being isolated within the northeaster to midwestern regions of the United States (Shen, Mead, \& Beard, 2011). Additionally, it is also the most common vector-borne illness within the United States (Tilly, Rosa, \& Stewart, 2008). Even still, Lyme disease is one of the hardest parasitic diseases to be diagnosed within humans.

Borrelia burgdorferi, like many other spirochetes, cannot usually be detected within the human body because the spiral parasites can tunnel into tissues in the body in concentrations that are often not detectable. As a result, current common tests for Lyme disease detect antibodies that the body commonly produces in response to acquiring this disease. However, since these tests only investigate responsive antibodies, they can only be detected as early as 2-3 weeks after infection, which is often too late to avoid many symptoms and side effects of the disease. Additionally, without treatment, the later stages of Lyme disease can cause neurological and cardiovascular symptoms that are very hard to diagnose and treat. Thus, there is a need to quickly and efficiently test for Lyme disease so that early treatment can help avoid the debilitating symptoms caused by this disease. 


\section{Engineering fundamentals of problem}

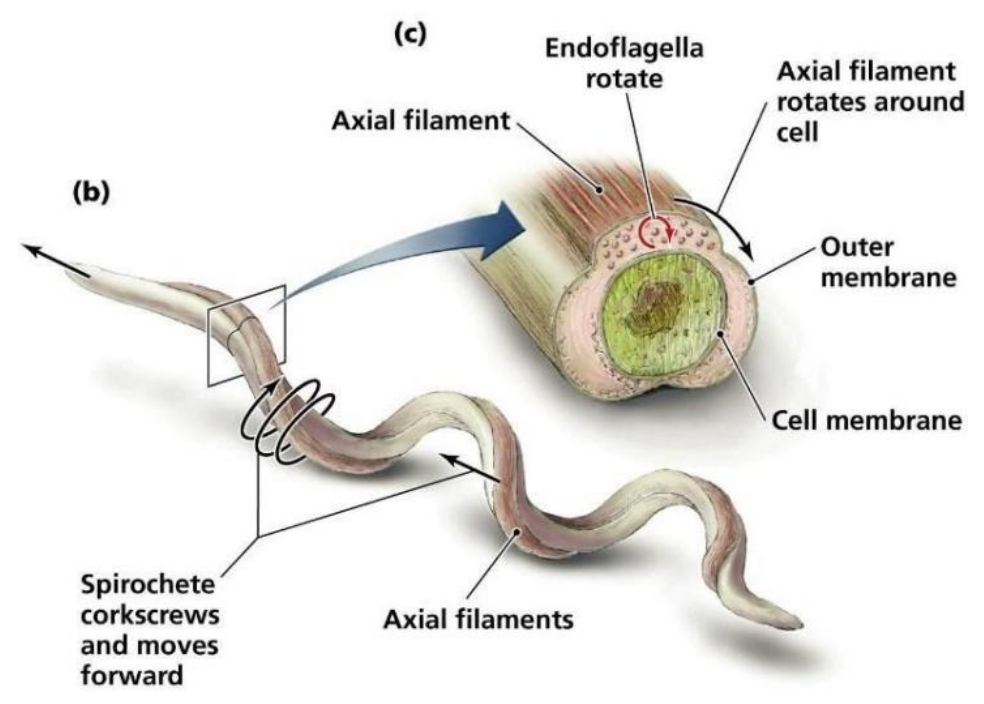

Figure 1: Characteristics of Phylum Spirochaetes

Source: https://drjeffreyjhuntnaturopathicphysician.com/category/lyme-disease/

Borrelia is the genus of eubacteria that is responsible for Lyme disease. It is a part of the phylum Spirochaetes, which also includes the agents that cause syphilis and relapsing fever. Bacteria within this phylum are notable for their spiral body with flagella found inside an outer membrane (Tilly et al., 2008). A visual representation of this unique bacterial structure can be seen in Figure 1.

For the most part, only three of these bacteria species collectively known as Borrelia burgdorferi sensu lato, cause Lyme disease: including sensu strictu, garinii, and afzelii (Tilly et al., 2008). While the symptoms resulting from these different species correspond, sensu strictu, the only species found in the United States, is associated more strongly with joint pain, while the others are known for neurological symptoms and skin rashes, respectively.

Borrelia burgdorferi is a tick-borne parasite commonly found in small mammals. While the normal reservoir of hosts does not experience the symptomatology associated with Lyme disease, when other animals such as humans or dogs are infected, the immunopathological response to this bacterium results in Lyme disease. Humans are infected with Borrelia 
burgdorferi when an infected tick, usually in the small nymphal stage, bites a human and remains attached for around 24-36 hours. This period provides enough time for the Borrelia burgdorferi living in the tick's midgut to transfer into the salivary glands and onto the skin of the human (Tilly et al., 2008). From there, Bb can enter the skin and infect the human.

Most bacterial diseases affect humans through virulence factors, or molecules specifically made by the bacterium to attack hosts in order to survive; however, Borrelia burgdorferi is divergent in that it does not produce extraneous virulent molecules (Tilly et al., 2008). Instead, the normal chemicals created by Borrelia burgdorferi for survival and reproduction are the same molecules that cause Lyme disease. These molecules can be sorted into two groups: those that play a role in the initial infection and those that contribute to long-term survival of the parasite. To initially infect a host, outer surface proteins (Osp) within Borrelia burgdorferi known as OspA and OspC are important. OspA is a binding protein for Borrelia burgdorferi that attaches it to the gut of the tick (Wagner, et al., 2012). When exposed to the salivary glands of the tick, OspA disappears and stops being produced so that there is more room for OspC. OspC then spreads across the skin of the human to establish a new colony (Tilly et al., 2008). OspC helps the Borrelia burgdorferi colonize across the skin of a human. This is what initially creates the rash, known as erythema migran, emblematic of Lyme disease. By first colonizing as a rash on the human's body, $\mathrm{Bb}$ is able to avoid immune responses during initial infection from a tick. After initial infection, the Borrelia burgdorferi bacteria travel into the bloodstream and other tissues in the body. Here, vIsE- meaning variation of its surface epitopes- and other proteins are produced that surround $\mathrm{Bb}$ to help establish a persistent infection within the new host and resist immune responses (Tilly et al., 2008). Research has shown that vIsE is integral to the infection of humans with Lyme disease (Rogovskyy \& Bankhead, 2013). Bb is known to cause a gene conversion through an unknown mechanism, which constantly produces antigenic variation within vIsE (Brisson, Drecktrah, Eggers, \& Samuels, 2012). This enables Bb to evade the immune system, since immune systems are constantly fighting a new antigen surface that is genetically variable from others. This antigenic variation also means that people with Lyme disease can be reinfected with $\mathrm{Bb}$ found in the body since the genetic variation of the surface proteins causes it to biologically be considered foreign. 
Several pathophysiological reasons explain why Borrelia burgdorferi is so detrimental within the human body and why it is so hard to treat. Firstly, while Borrelia burgdorferi can make certain proteins, they are chemoheterotrophic. This means Bb lack common mechanisms for creating carbon-based molecules including nucleotides, various acids, and enzyme cofactors (Tilly et al., 2008). As a result, the nutrients required for the parasite to survive must be taken from the human host. Borrelia burgdorferi's spiral shape and corkscrewing movement style allows it to easily travel throughout the body and tunnel into tissue too dense for most molecules to travel. Therefore, $\mathrm{Bb}$ can be found in almost every tissue type in the body, including: fibroblasts, lymphocytes, synovium, endothelium, neurons, and glial cells (Franz, et al., 2001). Being found throughout the body in various cell types helps the $\mathrm{Bb}$ to avoid the immune system and antibiotic treatment since most defenses against infectious diseases cannot target so many different types of tissues. Additionally, Borrelia burgdorferi's ability to burrow deeply into tissues often means it is also out of reach of almost all antibiotics and immune response organisms. Finally, whereas most bacteria cannot survive within these deep tissues since there is less of a supply of hemoglobin in such areas, $\mathrm{Bb}$ does not require iron to survive, and can, thus, theoretically live inside these dense tissues indefinitely. These pathophysiological traits are generally believed to be the cause of recurrent, or chronic Lyme disease.

Borrelia burgdorferi traveling to each of these tissue types result in the various, wideranging symptoms of Lyme disease. For example, the typical joint pain and arthritis associated with Lyme disease results from the $\mathrm{Bb}$ traveling into the joints- tendons and synovium around joints. Neurological symptoms: Bell's Palsy, demyelination, memory loss, lack of concentration, and headaches, come about when the Borrelia burgdorferi travels into neuronal tissues and the cerebrospinal fluid. Traveling into cardiac tissue, as well as through endothelium, can contribute to heart palpitations, irregular heartbeats, and hypertension. Finally, when $\mathrm{Bb}$ travels into muscular tissues, muscle weakness or numbness may occur.

Furthermore, a mixture of linear and circular plasmids can be found within $\mathrm{Bb}$. While researchers are unsure of the benefits of having both plasmid types (Tilly et al., 2008). It is believed the circular plasmids help minimize interactions within the body when $\mathrm{Bb}$ is in hiding 
deep within tissues (Tilly et al., 2008). Finally, while the chromosomes within Borrelia burgdorferi barely reproduce, the plasmids are constantly dividing and mutating. As a result, while infected ticks all have similar Bb bacteria, differences in the sequence of the genes make it hard for one to use genetic markers within the plasmid DNA for identification (Tilly et al., 2008). This makes it even harder to treat Lyme disease, since many antibiotics target specific genes found in specific regions of the infectious bacteria.

Three separate stages of Lyme disease are generally recognized. Early localized Lyme disease begins at infection and lasts for approximately four weeks. Symptoms associated with this stage include the Erythema migran rash that occurs, according to the CDC, in $60-80 \%$ of diagnosed cases (Center for Disease Control). Other symptoms include muscle or point pain, fatigue, headaches, and fever (Center for Disease Control). However, it is possible that no symptoms are observed in this stage. The next stage of Lyme disease is considered Early disseminated infection- when the infection begins to spread throughout the body. This occurs from one to four months. Symptoms generally include dissemination of Erythema migran rash, followed by clearing, muscle weakness or numbness, joint swelling or pain, heart palpitations, headaches, fainting, poor memory, and lack of concentration (Healthwise Staff, 2014). Finally, chronic Lyme disease, sometimes considered recurrent Lyme disease, occurs anywhere from five months to year after infection. Symptoms related with this stage of Lyme disease can occur in patients diagnosed with Lyme disease even when serological testing do not identify the presence of $\mathrm{Bb}$. Symptoms include arthritis, lack of concentration, demyelination of the myelin sheath, malaise, Bell's Palsy, irregular heart rhythms, trouble sleeping, and memory problems (Center for Disease Control).

Lessons from prior responses to the problem

While there are currently vaccines for dogs to prevent Lyme disease, there are no current vaccines for humans. In 1998, a vaccine for Lyme disease called LYMErix ${ }^{\mathrm{TM}}$ was tested and approved for use by the United States Food and Drug Administration approved. It works by providing patients with a small amount of recombinant OspA over three shots at zero, one, and twelve months (Group, 2006). Investigation revealed that after the three doses, $76 \%$ of 
symptomatic and $100 \%$ of asymptomatic Lyme disease was prevented through the use of this vaccine (Group, 2006). However, after 4 years, the vaccine was removed from market due to bad sales after rumors of adverse side effects (Poland \& Jacobson, 2001). Recent research has revealed that this vaccine most likely is not as potent as initially thought, since the vaccination did not help prevent even the most common mutations of the bacteria (Tilly et al., 2008). As described above, since Bb's genes mutate relatively quickly, it is hard to target a genetic sequence with a vaccine. Similarly, while the vaccination given in a flu shot is updated every year, changes in genetic code and new strands of the flu cause this vaccination to often not be very effective.

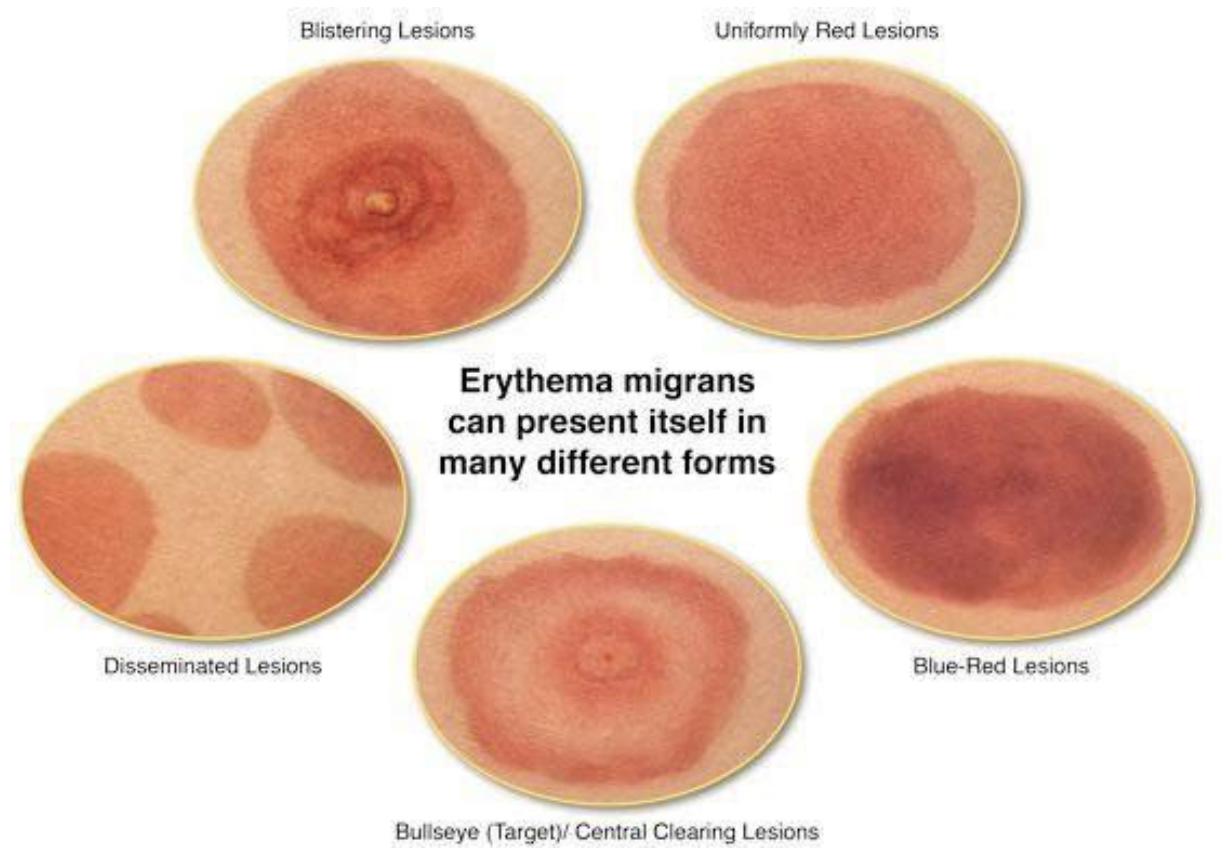

\section{Figure 2: Different Forms of Erythema Migran Rash}

\section{Source:}

https://www.science-art.com/image/?id=5799\&pagename=Erythema_migran_Rash_Types\#.Ws UlnpeQxPY

In terms of testing for Lyme disease, there have been very few techniques used to diagnose someone with Lyme disease. In 1975, Lyme disease was first established as a condition based on symptomatology. One of the most prevalent symptoms of early-stage Lyme disease is a 
rash known as Erythema migran (EM). While this rash comes in several forms as shown above in Figure 2, it is generally characterized as a warm, non-itchy rash. While these rashes are a good early indication that $\mathrm{Bb}$ is present, these various rash types closely resemble other rashes and can be misdiagnosed. Additionally, not all patients experience a rash. Other common symptoms and physical findings used to diagnose Lyme disease include arthritis-like aches, exposure to a tick, and cold-like symptoms.

Most diagnostic tests for Lyme disease do not test for the Borrelia burgdorferi, but antibodies that result from Lyme disease. Tests that detect $\mathrm{Bb}$ bacteria instead of antibodies to Lyme disease are not common since Borrelia burgdorferi cannot be identified through normal viral testing. For example, while most bacterial infections are cultured by taking a swabbed sample, $\mathrm{Bb}$ cannot be characterized through culturing. Additionally, since $\mathrm{Bb}$ is often not found in high enough concentrations in any one place in the body, blood or tissue sample often also cannot characterize $\mathrm{Bb}$ and Lyme disease. 
Tier 1: IgG or IgM EIAs (combined or separate)

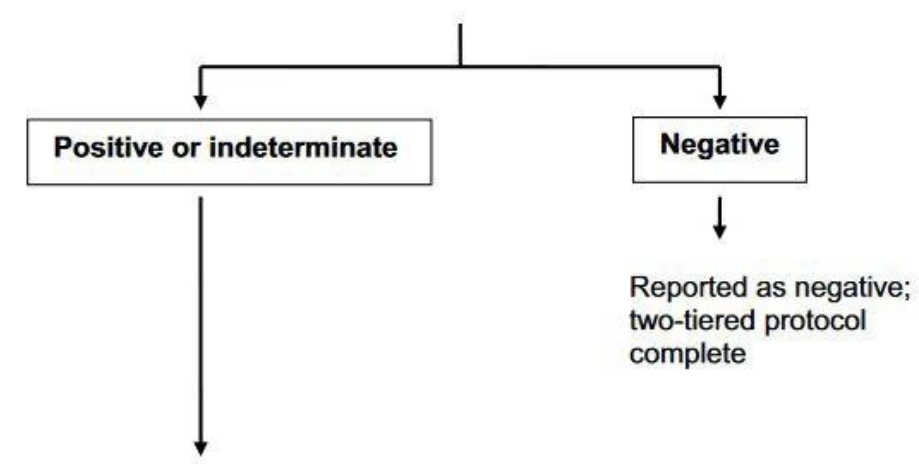

Tier 2: IgG and/or IgM immunoblots (separate)

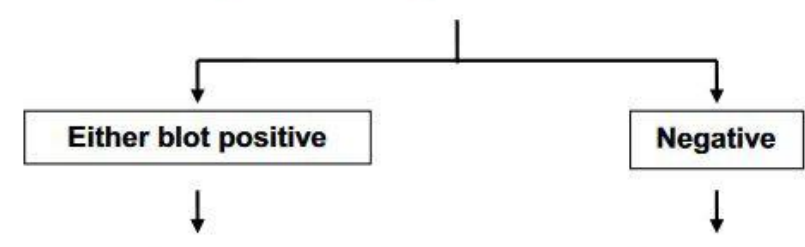

IgG-positive reported as positive

Reported as

IgM-positive reported as positive negative

BUT clinically relevant only in

early disease of less than 1 month

duration

\section{Figure 3: System of Serological Tests Used in Diagnosing Lyme disease}

Source: Laboratory diagnostic testing for Borrelia burgdorferi infection by B. J. Johnson

In 1995, the NIH and the FDA worked together to create a standard procedure to diagnose Lyme disease (Johnson, 2012). They created a two-part series of testing using serologic tests that is outlined above in Figure 3. While other diagnostic tests for Lyme disease exist, only this system of serological tests is approved by the FDA and used outside of academia. Within the first tier, a blood sample is gathered from a patient and analyzed with serological enzyme immunoassays (EIAs) that detect antibodies commonly found when a body is first exposed to an antigen- immunoglobulin $\mathrm{M}(\mathrm{IgM})$ and/or immunoglobulin $\mathrm{G}$ (IgG) (Johnson, 2012). If these EIAs show a concentration of antibodies within the body that is less than the range of a Lyme disease patient, the test is considered negative and further testing is not required. However, when the EIAs are within the range of test values for Lyme disease, the serological test can be either considered positive or indeterminant. Indeterminant occurs when a test value is found where the 
range of test values for Lyme disease patients and the normal populace overlap. When either a positive value or an indeterminant value are found, the second tier of testing is done. The second tier involves blot testing the blood, usually using a technique called the Western Blot. If both tests show positive results, then the patient is positively diagnosed with Lyme disease.

While this two-tier system has a specificity of around $98 \%$, it only has about a $42 \%$ sensitivity (Molins, Ashton, \& Belisle, 2015). This means that while the test is good at diagnosing Lyme Disease from other diseases, it is only correctly diagnosing about 4/10 cases of Lyme Disease. Even still, this system is beneficial because it utilizes a normal blood tissue sample, which does not require an Erythema migran rash to test. Additionally, such a blood test is common within medical laboratories, takes about 3 days to complete, and produces no extremely dangerous waste. In terms of cost, since multiple blood tests are used for diagnosis of the sample, this testing system costs about $\$ 500$, which is expensive for a such diagnostic tool (ThermoFisher Scientific).

\section{Project objectives and constraints}

The goal of this assessment is to find a more accurate test for early stage Lyme disease. The various individual goals for the ideal alternative diagnostic test are listed below:

- Have a sensitivity greater than or equal to $85 \%$

- Have a specificity greater than or equal to $95 \%$

- Able to be performed whether an Erythema migran rash is present or not

- Able to be tested at a regular medical laboratory with no additional equipment

- Be able to be diagnosed within 3 days

- Cost no more than $\$ 500$ a test which is approximately the cost of the current two-tier serologic system in place

- Have no extremely dangerous waste products

There are many limitations in finding improved techniques to diagnose Lyme disease within its early stages of infection from week 0 to week 3 . Within this period, serologic tests commonly used to diagnose Lyme disease cannot be used since not enough antibodies to fight the $\mathrm{Bb}$ are present in the body this early. The most common type of diagnostic test used for this stage of 
infection includes observation and symptom-based diagnosis. However, the symptoms of early stage Lyme disease mostly resemble that of the common cold or Chronic Fatigue Syndrome, which makes it hard to definitively diagnose within biological testing. Additionally, while over $60 \%$ of patients experience a form of rash form Lyme disease, these rashes can often be overlooked as other skin problems such as poison ivy or eczema. Even worse, another portion of patients do not experience a rash. In this case, Lyme disease is almost never considered as a likely cause of its initial symptoms, and thus results in improper treatment.

Besides biological constraints, another large factor for this diagnostic test is time. The longer Lyme disease remains untreated, the more time $\mathrm{Bb}$ has to infect the body. As a result, the diagnostic treatments should be able to ascertain a diagnosis within a few days so that the longterm effects of Lyme disease may be avoided. 


\section{Candidate Solutions}

This section reviews viable testing options to diagnose Lyme disease other than what is currently on the market. This analysis entertains numerous solutions, even though it mainly focuses on the three most viable options.

Scope of solutions considered

Countless solutions were contemplated. Through review, the three best solutions are explained below, however, other solutions, including non-engineering solutions and historical solutions are worth mentioning for contextual purposes. One solution considered was simply publicizing what Lyme disease is and why there is a necessity to accurately diagnose it within its early stages within the medical community. While this problem would help increase the number of cases of Lyme disease, it does not help improve the accuracy of testing, especially within early stage Lyme disease. Another solution that was thoroughly researched was cell culturing. Cell culturing is used to identify countless bacterial infections within humans simply through swabbing of mucus membranes, or blood samples. However, while there is a culturing process for Borrelia burgdorferi, a specific culture medium is required called Barbour-Stoenner-Kelly since $\mathrm{Bb}$ is chemoheterotrophic and cannot form identifiable nucleotides of more commonly used culturing mediums (Wang, Aguero-Rosenfield, Wormser, \& Schwartz, 2010). Additionally, the incubation period for such a culture is approximately 12 weeks since the reproduction period on $\mathrm{Bb}$ is 7-20 hours (Wang et al., 2010). Consequently, culturing is not a viable option for identifying Lyme disease since the time period is too long and the materials necessary are uncommon. Finally, direct microscopic detection systems were considered, including dark-field microscopy and direct microscopy. While microscopic inspection of a sample is possible, human tissue does not contain enough bacteria for this to be effective (Wang et al., 2010). Microscopic techniques are very common for identification of $\mathrm{Bb}$ within ticks, however. A third solution considered was flow cytometric borreliacidal antibody test (fcbat). While this test is highly specific towards Borrelia burgdorferi, the test requires viable spirochetes which are not always present (Jobe, Lovrich, \& Callister, 2008). Additionally, interference can occur between 
microbes and the test requires significant amounts of technical ability (Jobe et al., 2008). Thus, this test was out-ruled due to its relative difficulty to perform.

\section{Explanation of candidate solutions}

The three candidate solutions chosen for further comparison include polymerase chain reaction detection, metabolic biosignature determination, and further serological testing.

\section{Polymerase Chain Reaction (PCR) Detection}

Polymerase Chain Reactions uses polymerase, a thermostable enzyme, to amplify monomer DNA or RNA chains and form polymer chains of these structures that can then be identified (American Association for the Advancement of Science, 1988). PCR is very popular in molecular biology since even small tissue samples can produce readable DNA chains (American Association for the Advancement of Science, 1988). The relative cost to a patient for such test would be about $\$ 400-500$ including a skin biopsy, storage, transportation, and testing (Matthews, 2011).

\section{Process for testing}

Numerous sample tissues can be used to diagnose Lyme disease in humans including skin biopsy samples from Erythema migran, blood/plasma, cerebrospinal fluid, and synovial fluid (Wang et al., 2010). Generally, synovial fluid is used to identify those with Lyme Arthritis and cerebrospinal fluid is used for those with chronic neurological symptoms. For those with a rash, skin sampling is easier and more effective than blood or plasma samples; however, for those that do not experience a rash, a blood sample can be used. Additionally, several techniques can be used when running a polymerase chain reaction for Lyme disease detection including standard PCR, nested PCR, competitive PCR, and real-time PCR (Wang et al., 2010). These PCR techniques simply vary in the primer sets used and the type of data gathered.

PCR requires the use of a specific PCR machine called a thermal cycler. Due to the popularity of PCR in clinical testing, PCR machines are common in clinical laboratory settings. Mechanistically, PCR works applying helicase enzymes and heat to a sample of DNA; this opens the double helix into two separate strands (American Association for the Advancement of 
Science, 1988). Next, a primer is added to the cooled sample which works to expose DNA base pairs and prime for copying (American Association for the Advancement of Science, 1988). Various primers are used which depend on the type of PCR being ran and the desired results. In standard PCR, the ideal primer for identification of Borrelia burgdorferi that amplifies every base pair 100-300 times (Wang et al., 2010). In other PCR variations, two primers can be used in series or concurrently to produce similar results. Finally, polymerase enzyme is used to polymerize, or multiply, the DNA base pair strands (American Association for the Advancement of Science, 1988). Figure 4 below shows the process of this polymerization. One DNA strand generally creates hundreds of thousands of copies within this reaction.

\section{PCR: Polymerase Chain Reaction}

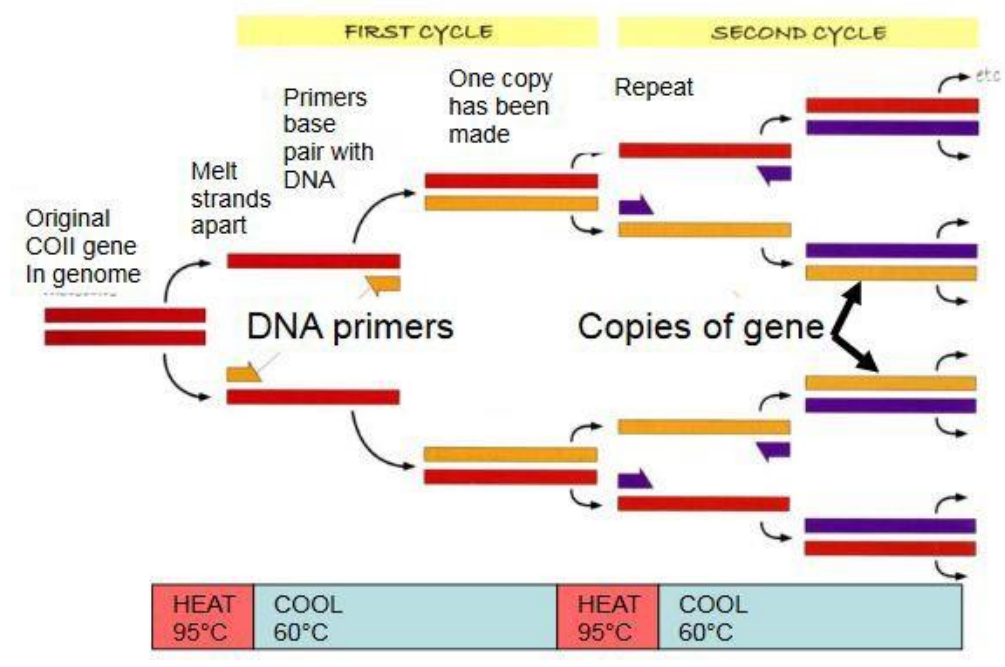

\section{Figure 4: Polymerase Chain Reaction Scheme}

Source: American Association for the Advancement of Science, 1988

After the reaction occurs, the experimental sample is compared to control DNA strands found in Borrelia burgdorferi, including chromosomally encoded genes as well as plasmidencoded genes such as OspA (Wang et al., 2010). These identify whether the DNA strands isolated were $\mathrm{Bb}$ or not. Waste products of PCR include PCR liquid waste and excess skin biopsy materials. These materials are relatively safe and require only normal disposal (American Association for the Advancement of Science, 1988). 


\section{Testing Challenges}

PCR testing requires significant training to yield proper results especially since the concentration of Borrelia burgdorferi in human tissues is low and hard to isolate. As Wang explains in Borrelia: Molecular biology, host interaction, and pathogenesis:

While many laboratories have the necessary materials required for testing, the variability of adequate personnel training would result in additional variability in results than the percentages provided below. Additionally, degradation of $\mathrm{Bb}$ during transport, storage, and testing cause variability in results. In attempts to combat this degradation, specimen stored for a long period of time are fresh frozen to yield higher yields when testing. Also, if the tissue sample is left in the testing medium for over 24 hours, some Borrelia burgdorferi may travel from the sample into the medium. Consequently, the medium must be placed in the PCR as well, to ensure correct results. Finally, while blood samples are the easiest samples to obtain, blood contains PCR inhibitors, especially when antibiotics are present within the body, that greatly reduce the effectivity of PCR analysis of such samples. (Wang et al., 2010)

As described, PCR is only effective when testing Erythema migran skin samples. Additionally, it would require significant standardization before use.

\section{Effectivity}

The table below summarizes the accuracy of PCR testing of various tissues across multiple studies. Sensitivity is a fractional measure of positive test results over the amount of people tested with Lyme disease while specificity is the percentage of people who have Lyme disease that tested positive for it.

Table 1: Effectivity of PCR testing of Various Clinical Specimen

\begin{tabular}{|l|l|l|l|}
\hline Clinical Specimen & No. of Studies & Median Sensitivity (\%) & $\begin{array}{l}\text { Reported Specificity } \\
(\%)\end{array}$ \\
\hline
\end{tabular}




\begin{tabular}{|l|l|l|l|}
\hline $\begin{array}{l}\text { Skin Biopsy, } \\
\text { Erythema Migran }\end{array}$ & 17 & $69(36-88)$ & $98-100$ \\
\hline $\begin{array}{l}\text { Blood/Plasma/Seru } \\
\text { m }\end{array}$ & 10 & $21(0-100)$ & -- \\
\hline Cerebrospinal fluid & 18 & $32(5-100)$ & $93-100$ \\
\hline Synovial Fluid & 8 & $78(42-100)$ & 100 \\
\hline
\end{tabular}

Source: Detection of Borrelia burgdorferi, 2010

\section{Metabolic Biosignature}

In 2015, a group of researchers tested blood samples from hundreds of patients diagnosed with Early Lyme disease and many other diseases to identify specific early stage molecular features that could be used to diagnose someone with Lyme disease (Molins et al., 2015). While this is similar to serological tests, metabolic biosignature tests detect pre-immune response metabolites to Borrelia burgdorferi. Metabolites tested include peptides and several low-mass polyunsaturated fatty acids and lipids associated with in nervous tissue health, cholesterol formation, prostaglandin hormones dispersion, and cardiovascular enrichment (Molins et al., 2015). Together, this specific sequence of metabolic markers provides a fingerprint to identify early cases of Lyme disease.

These tests can be analyzed using liquid chromatography- mass spectrometry (LC-MS), a relatively common medical lab machine that separates substances based on polarity and mass to charge ratio (Molins et al., 2015). Similar to a serological test, such a biological test would cost about \$150-200 (Matthews, 2011).

\section{Process for Testing}

Metabolic biosignature testing uses blood samples as its clinical specimen. From the blood, sera- the protein rich liquid that separates from coagulated blood- are collected after centrifugal and extraction separation (Tuck, Chan, \& Brenner, 2009). Next, the sera are extracted with methanol to isolate metabolites from other sera particles through a series of methanol 
solvent extractions (Molins et al., 2015). This sample is then collected and analyzed with the LCMS which separates metabolites based on retention time and mass (Molins et al., 2015). This data is then compared at 44 metabolite biomarkers that differ between those who have Earlystage Lyme disease and those who do not (Molins et al., 2015). Computer analysis of various concentrations of each of the biomarkers between a sample and the two controls- ideal positively and negatively diagnosed examples- evaluates the sample to provide a diagnosis. Waste products of metabolic biomarker testing include excess blood sample materials and extraction liquids, both of which do not pose a great risk.

\section{Testing Challenges}

While LC-MS machines are common for urine drug testing, there is a possibility that certain laboratories would not have this piece of equipment. Therefore, the time frame in which to receive test results may grow from a 2-3 day service to 4 or more days. Additionally, using biomarkers is a relatively new diagnostic technique. Since the computer is better able compare the biomarkers between the control and product, no human comparison is necessary for evaluation of these results. Consequently, less training is required of laboratory specialists. Still, proper technique of serum extraction and machine usage is necessary. The samples must also be kept at a stable temperature below the heat inactivation temperature of $56^{\circ} \mathrm{C}$ to guarantee an accurate diagnosis.

Even though this form of testing seems promising, as a new field of study, the use of Liquid Chromatography- Mass Spectrometry is not standardized yet (Molins et al., 2015). Strict standardization would be required before use of this technology to ensure accuracy within the results. Otherwise, variability between lab procedures would cause further variation in results.

\section{Effectivity}

As a new form of identification, little research has been conducted on this diagnostic approach with only one source published. However, during this research project, over 450 blood samples were analyzed after identification of the metabolite biomarkers where an average of $89 \%$ of samples with Lyme disease and an average of $95 \%$ of people that did not have Lyme's disease were correctly diagnosed (Molins et al., 2015). 


\section{Synthetic OspC based Serology}

While serological testing targeting $\operatorname{IgM}$ and $\operatorname{IgG}$ is currently used to diagnose one with Lyme disease, serological tests that test for other immune responses can also be used to diagnose Lyme disease. However, during early infection with Lyme disease, while these normal immune chemicals are not present, specific immune cells targeting OspC are present. These can be tested in synthetically activated serological testing.

Such serological tests are analyzed in the same way the other EIAs were analyzed, using mass spectrometry as the main testing equipment (ThermoFisher Scientific). This test would cost approximately $\$ 180-250$, which is more expensive than the other serological tests because this test requires the synthesis of a peptide for analysis.

\section{Process for Testing}

The procedure for this test first requires the synthesis of a synthetic peptide epitope for OspC named OspC7 which can be done with an automated synthesizer (Jobe et al., 2008). Fluorescent enzymes are also bonded to OspC7 so that when an OspC protein is immobilized, the absorption can be measured through mass spectrometry (ThermoFisher Scientific).

The blood samples taken from patients are extracted as described above using for metabolic biosignature testing so that only sera remain. Then, the plates used for the reaction were primed with buffer washes and incubations. Then, the OspC7 was added which would then bind with solid surface proteins (ThermoFisher Scientific). The separated serum was added last to the solution and left to incubate for an hour (Jobe et al., 2008). A pictorial representation of this binding system is shown below in Figure 5. Silver acts as a solid phase stabilizer that makes the OspC7 stationary. Then, OspC within the sera binds to the OspC7 which causes a substrate to not absorb blue light, thus presenting a blue hue and allowing for easy identification by mass spectrometry. 


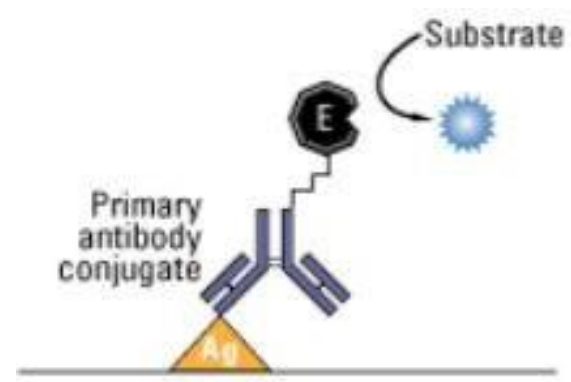

Direct Assay

\section{Figure 5: Binding System for Serological EIA Testing}

\section{Source: ThermoFisher Scientific, n.d.}

Then the microtiter plates were evaluated in a mass spectrometer at $490 \mathrm{~nm}$ of light (Jobe et al., 2008). Comparison of absorption values to those of controls then aids in the diagnosis of the patient. Waste materials include microtiter plates, extraction liquids, non-sera blood sample particles, and mass spectrometry liquid waste, all which is relatively safe waste materials (Jobe et al., 2008).

\section{Testing Challenges}

Most of the challenges concerning OspC7 serology testing reside in the difficulties of making the OspC7. Synthesis of OspC7 requires knowledge of how to use such equipment and additional purity testing. As a result, testing requires a lot more materials than normal serological testing. While this would mean that the whole testing process is longer than others, since the OspC7 can be synthesized and stored prior to usage, this test will also only take about three days to process. Additionally, while the test is more accurate than other serologies and OspC produces a considerable immune response, variations in sequencing of $\mathrm{OspC}$ within $\mathrm{Bb}$ make it hard to diagnose within all cases of Lyme disease (Wang et al., 2010).

\section{Effectivity}

Evaluation of this diagnostic technique only includes one experiment consisting of 157 test subjects tested a total of three times (Jobe et al., 2008). It was found that $73 \%$ of people with 
Lyme disease were appropriately diagnosed with Lyme disease while $98 \%$ of people without Lyme disease were diagnosed correctly as well (Jobe et al., 2008).

\section{Comparative assessment of Candidate Solutions}

Table 2 compares the three alternative solutions based on important determination factors. For PCR testing, due to the wide variations within results, only the statistics for skin biopsy of an Erythema migran rash will be shown below since it is the best sample used for identification of Early Lyme disease. Unfortunately, not everyone gets the rash, so the values shown below for PCR testing only represent a fraction of patients that show evidence of a rash.

Table 2: Comparative Analysis of Candidate Solutions

\begin{tabular}{|c|c|c|c|}
\hline & $\begin{array}{l}\text { Polymerase Chain } \\
\text { Reaction }\end{array}$ & $\begin{array}{l}\text { Metabolic } \\
\text { Biosignature }\end{array}$ & $\begin{array}{l}\text { Synthetic OspC based } \\
\text { Serology }\end{array}$ \\
\hline Sensitivity & $69 \%$ & $89 \%$ & $73 \%$ \\
\hline Specificity & $99 \%$ & $95 \%$ & $98 \%$ \\
\hline Require rash & Yes & No & No \\
\hline $\begin{array}{l}\text { Sample from } \\
\text { Patient Required }\end{array}$ & $\begin{array}{ll}\text { Skin Biopsy } & \text { of } \\
\text { Erythema Migran } & \end{array}$ & Blood Sample & Blood Sample \\
\hline $\begin{array}{l}\text { Equipment } \\
\text { Required }\end{array}$ & PCR Machine & $\begin{array}{l}\text { Liquid } \\
\text { Chromatograph/Mas } \\
\text { s Spectrometer }\end{array}$ & $\begin{array}{l}\text { Automated synthesizer, } \\
\text { Mass Spectrometer }\end{array}$ \\
\hline Waste materials & $\begin{array}{l}\text { Skin biopsy materials, } \\
\text { PCR liquid waste }\end{array}$ & $\begin{array}{l}\text { Non-sera blood } \\
\text { sample particles, } \\
\text { extraction liquids }\end{array}$ & $\begin{array}{l}\text { Non-sera blood sample } \\
\text { particles, extraction } \\
\text { liquids, microtiter } \\
\text { plates, and MS liquid } \\
\text { waste }\end{array}$ \\
\hline
\end{tabular}


Maneto: The Temple University Multi-Disciplinary Undergraduate Research Journal | 3.1

\begin{tabular}{|l|l|l|l|}
\hline Skills required & $\begin{array}{l}\text { PCR } \\
\text { equipment/reading } \\
\text { training }\end{array}$ & $\begin{array}{l}\text { Input LC-MS } \\
\text { knowledge }\end{array}$ & $\begin{array}{l}\text { Synthesis and Mass } \\
\text { Spectrometry } \\
\text { knowledge }\end{array}$ \\
\hline Cost & $\$ 450$ & $\$ 175$ & $\$ 190$ \\
\hline Relative Time & 3 days & $3-5$ days & 3 days \\
\hline
\end{tabular}




\section{Project Recommendations}

After comparison of the three candidate solutions, this section reviews one recommended solution and the process required to put this solution into common practice.

\section{Recommended solution}

While PCR analysis and OspC7 serological testing are good alternatives to the current system for diagnosing Early Lyme disease, the best diagnostic test solution is metabolic biosignature testing. This form of diagnostic testing produced the highest sensitivity to patients with Lyme disease, did not require one to have an Erythema migran rash, was less costly than all other tests considered, and did not form any harmful waste. However, the specificity was lower than other solutions considered. Also, it required the use of a liquid chromatograph/ mass spectrometer, which might not be commonly found in a lab. This would cause testing to take one to two days longer than normal. However, testing involving liquid chromatography followed mass spectrometry is regular and becoming increasingly more common. LC-MS is used in commonly on clinical samples in toxicology, pharmacology, and therapeutic drug monitoring (Abou-Diwan, 2011). Recently, though, LC-MS has begun to be used in protein detection and endocrinology- such as the metabolic biocompatibility testing used above (Abou-Diwan, 2011). Consequently, it is assumed that LC-MS will become more common as time progresses, thus decreasing the relative testing time frame.

\section{Design and implementation challenges}

Several design and implementation hurdles must be overcome before this diagnostic alternative can be carried out.

\section{Implementation path}

While this path does not need to be followed perfectly, this section outlines the steps required to implement this diagnostic tool. Firstly, while metabolic biosignature testing has become common over the last five years, very little research has gone into its applications in diagnosing Lyme disease, with only one research paper specifically detailing the use of such diagnostic tools for characterization of Lyme disease. Accordingly, additional research would 
have to be conducted to prove the validity of the diagnostic tool. This would most likely take approximately 1,000 additional testing samples since many anomalies are usually only found after a large amount of data is collected.

Once biosignature for Lyme disease is validated through additional research studies, the National Institute of Allergy and Infectious Diseases, NIAID, which would under the National Institutes of Health, NIH, would have to also perform clinical trials and research the validity of the diagnostic tool. Similar to other clinical trials, such research would have to be reviewed by the Institutional Review Board throughout the research process to ensure the safety of all involved (Health, 2017). This specific research project would also help the NIH set forth standards for the testing. These standards will define what is considered a positive and negative test result and provide controls for both examples.

After the determination of standard testing procedures, the NIH would then publicize the test as a newly approved diagnostic tool for Early Lyme disease. From here, hospitals would have to adopt this new system and teach staff members how to properly perform the test and diagnose patients. Since numerous other tests require the use of blood samples, most likely the only additional training would possibly be the use of a Liquid Chromatograph-Mass Spectrometer and details on how to read the results.

\section{Possible Complications}

Several possible complications may hinder the implementation of metabolic biosignature testing. One such difficulty may be lack of resources to perform further testing. Research requires a significant amount of funding, especially when collecting and analyzing hundreds to thousands of human blood samples. However, several foundations exist to help fund Lyme disease research, including the Global Lyme Alliance, the Lyme disease Association, and the American Lyme disease Foundation. Additionally, as of 2017, the NIH is already sponsoring this research in attempts to improve diagnostic techniques, so this possible complication has been bypassed (National Institute of Allergy and Infectious Diseases, 2017).

Another possible complication is further testing not corroborating with the original research findings. This difficulty would require additional research to explain both prior findings 
and determine the capacity in which such a metabolic test could work. If the test is found to be significantly inaccurate, research may even have to be stopped and other alternative solutions may have to be explored. Even still, this possibility is very rare as metabolic testing is considered an accurate detection system for early detection of many diseases, including ovarian and cervical cancer (Yang, et al., 2017). Additionally, in the research collected by Molins that formed the metabolic biosignature for Lyme disease, over 450 blood samples were taken from controls, people with known Lyme disease, those most likely with Early Lyme disease based on rash and symptoms, and those with other known diseases (Molins et al., 2015). Consequently, this data can be considered accurate with very low probability of major flaws.

Finally, in making any diagnostic test, the largest difficulty is determining a standard mark between tests that are considered positive and those that are considered negative. If there is too much variation in any one of the 44 biomarkers, a revision in which a biomarker is removed or revised may need to be made. This alteration can only come about with additional research and testing but will not significantly hurt the diagnostic test. Instead, with further refining, the metabolic biosignature testing can become even more accurate.

\section{Conclusion}

Implementing a new standard for the diagnosis of Early Lyme disease using metabolic biosignature testing would provide a plethora of benefits that outweigh the costs. First, with a more accurate and better applicable diagnostic tool for Early Lyme disease, people will be able to be diagnosed and treated for Lyme disease earlier. In effect, less cases of Chronic Lyme disease will occur since the time span before initial treatment plays a major role in the severity and duration of Lyme disease symptoms. This makes for a healthier public. With less people having to deal with the detrimental neurological and cardiovascular symptoms associated with Lyme disease, the hospital resources that would have gone in to treating these patients can be used elsewhere. Even still, hospital may have to pay for LC-MS equipment to run these tests. While this has no overt instant benefits, usage of this machine over time for many tests will defray these costs. 
Additionally, by establishing a more accurate system of Early Lyme disease detection, Lyme disease research can focus on other areas of interest, including studies on possible vaccinations, autoimmunity, and pathogenesis. Moreover, as a new technology, metabolic biosignatures will be publicized through their use in Lyme disease diagnostics. This will encourage interest in this new field of diagnostic testing and encourage companies to make additional diagnostic tests for hard-to-diagnose diseases similar to Lyme disease.

Overall, the application of this new diagnostic tool displays a lot of positive results with very few negative outcomes. As a result, this project is extremely worth doing for the health of all with the potential to contract Lyme disease. 
Glossary

Antigenic variation - mechanism used by infectious bacteria to alter its surface proteins and effectively avoid the immune response of the host.

Chemoheterotrophic - when an organism cannot manipulate organic substances, namely carbon, to survive. This means common biological matter used for survival including nucleotides and acids cannot be formed by these organisms.

Epitope - molecule or part of a molecule with which an antibody bonds

Eubacteria - bacteria that require only one cell to survive.

Flagella - tail like structures on certain bacteria that enable them to swim or move. Within spirochetes, the flagella are coiled around the body so that these bacteria move in a corkscrew-like motion.

Immunopathological - the immune responses of an organism that come about as a result of a pathogen or disease.

Lyme disease - a vector-based disease that results from human infection with Borrelia burgdorferi. Lyme disease is often characterized by an Erythema migran rash, arthritis, and neurological symptoms.

Parasite - an organism that survives by living off and harming another organism.

Pathophysiological - the physiological, or normal function, changes of an organism that come about from a certain pathogen or disease.

Polymerase - a group of enzymes that react with nucleic acids, namely DNA and RNA, to form long polymer chains available for identification of such nucleic acids.

Polymerase Chain Reaction - the use of polymerase to react with small nucleic acids- DNA and RNA- to form identifiable chain polymers. 
Plasmids - small structures of DNA that are usually circular and replicate under specific conditions separately from the general chromosomal DNA used for reproduction.

Sensitivity - ability of a diagnostic test to correctly diagnose those with a certain disease.

Serologic test - a blood test in which blood serum is separated from clotted blood to detect specific antibodies.

Species Complex - scientific classification of a group of species that are almost indistinguishable but contain slight differences in their genomes that differentiate them as separate species.

e.g. Within the Borrelia burgdorferi species complex, various species of Borrelia caused the same symptomology, but certain species were associated more heavily with certain symptoms, such as joint pain.

Specificity - the ability of a diagnostic testing to positively identify those that do not have a certain disease.

Vector - a living organism that can transfer an infection disease to humans.

Virulence factors - molecules, including toxins and enzymes, that certain organisms specifically produce to harm or hide from a host in order to survive. 


\section{References}

Abou-Diwan, C. (2011, June 1). LC-MS/MS in the clinical lab: Strengths, applications, challenges. Retrieved from Medical Labratory Observer: https://www.mlo-online.com/lc-ms-ms-in-the-clinical-lab-strengths-applications-challeng es.php

American Association for the Advancement of Science. (1988). PCR: The polymerase chain reaction. Marine Genomics, 487-491.

Brisson, D., Drecktrah, D., Eggers, C. H., \& Samuels, D. S. (2012). Genetics of Borrelia burgdorferi. National Institutes of Health Annu Rev Genet.

Center for Disease Control. (n.d.). Lyme Disease: What you need to know. Retrieved from Center for Disease Control: https://www.cdc.gov/lyme/resources/brochure/ lymediseasebrochure.pdf

Franz, J. K., Fritze, O., Rittig, M., Keyber, G., Priem, S., Zacher, J., . . Krause, A. (2001). Inisghts from a novel three-dimensional in vitro model of Lyme arthritis: Standardized analysis of cellular and molecular interactions between Borrelia burgdorferi and synovial explants and fibroblasts. Arthritis \& Rhumatology, 151-162.

Group, N. P. (2006). When a vaccine is safe. nature, 509.

Health, N. I. (2017, October 20). NIH clinical research trials and you: The basics. Retrieved from National Institutes of Health: https://www.nih.gov/health-information/nih-clinical-research-trials-you/basics

Healthwise Staff. (2014, June 4). Stages of Lyme Diseasae: Topic overview. Retrieved from WebMD: 338-034634-4054

Jobe, D. A., Lovrich, S. D., \& Callister, S. M. (2008). Significantly improved accuray of diagnosis of Early Lyme Disease by peptide enzyme-linked immunosorbent assay based on the Borreliacidal antibody epitope of Borrelia burgdorferi OspC. Clinical and Vaccine Immunology, 981-985. 
Johnson, B. J. (2012). Labratory diagnostic testing for Borrelia burgdorferi infection. In J. Halperin, Lyme Disease: An Evidence-based Approach (pp. 73-88). Center for Disease Control.

Matthews, L. (2011, August 17). Lyme Disease Test Cost. Retrieved from Lyme Disease: A practical guide: http://lymediseaseguide.net/lyme-disease-test-cost

Molins, C. R., Ashton, L. V., \& Belisle, J. T. (2015). Development of a metabolic biosignature for detection of early Lyme Disease. Clinical Infectious Diseases, 1767-1776.

National Institute of Allergy and Infectious Diseases. (2017). Current Efforts in Lyme Disease Research. Retrieved from National Institute of Allergy and Infectious Diseases: https://www.niaid.nih.gov/sites/default/files/NIAIDLymereport2015.pdf

Poland, G. A., \& Jacobson, R. M. (2001). The prevention of Lyme Disease with vaccine. Vaccine 19, 2303-2308.

Rogovskyy, A., \& Bankhead, T. (2013). Variable VlsE is critical for host reinfection by the Lyme Disease spirochete. Public Library of Science, 1-12.

Shen, a. K., Mead, P. S., \& Beard, C. B. (2011). The Lyme Disease vaccine: A public health perspective. Clinical Infectious Diseases, 247-252.

ThermoFisher Scientific. (n.d.). Overview of ELISA. Retrieved from Thermo Fisher Scientific: https://www.thermofisher.com/us/en/home/life-science/protein-biology/protein-biology-l earning-center/protein-biology-resource-library/pierce-protein-methods/overview-elisa.ht $\mathrm{ml}$

Tilly, K., Rosa, P. A., \& Stewart, P. E. (2008). Biology of infection with Borrelia burgdorferi. Infect Dis Clin North Am, 217-234.

Tuck, M. K., Chan, D. W., \& Brenner, D. E. (2009). Statndard operating procedures for serum and plasma collection: Early detection research network consensus statement . Journal of Proteome Research, 113-117. 
Wagner, B., Freer, H., Rollins, A., Garcia-Tapia, D., Erb, H. N., Earnhart, C., . . Meeus, P. (2012). Antibodies to Borrelia burgdorferi OspA, OspC, OspC, and C6 Antigens as markers for early and late infection in dogs. Clinical Vaccine Immunology, 527-535.

Wang, G., Aguero-Rosenfield, M. E., Wormser, G. P., \& Schwartz, I. (2010). Detection of Borrelia burgdorferi. In D. S. Samuels, \& J. D. Radolf, Borrelia: Molecular biology, host interaction, and pathogenesis (pp. 443-466). Norfolk: Caister Academic Press.

Yang, K., Bairong, X., Wang, W., Cheng, J., Yin, M., Xie, H., . . . Li, K. (2017). A comprehensive analysis of metabolomics and transcriptomics in Cervical Cancer. Scientific Reports, 50-62. 